\title{
Historia clínica informatizada: herramienta para la atención integral al paciente con enfermedad renal crónica
}

\author{
Judith Sánchez Torres
}

Hospital del Vinalopó. Alicante

\section{Introducción:}

Los pacientes afectados de enfermedad renal crónica en cualquiera de sus estadíos son pacientes crónicos, complejos, que requieren de una atención sanitaria global que, desde los diferentes puntos de vista sanitarios y sociales, sea capaz de atender las necesidades que se generen en sus procesos de salud-enfermedad. En la sociedad de las nuevas tecnologías, son muchos los programas informáticos que se han desarrollado para gestionar la historia clínica de los pacientes en centros sanitarios. En el caso de los pacientes nefrológicos, sobretodo en sus estadíos más avanzados en los que precisan de tratamiento renal sustitutivo, existen gran cantidad de aplicaciones para gestionar sus procesos de enfermedad, sin embargo, no suelen tener un punto de vista integral y no engloban todos los aspectos de la historia clínica, sin ofrecer la posibilidad de interactuar entre los diferentes profesionales encargados de su tratamiento. Por historia clínica global entendemos aquella que es capaz de integrar toda la actividad asistencial del o de los centros sanitario en los que se atiende a un paciente, fomentando la comunicación y el intercambio de datos entre profesionales, tanto de atención primaria como de especializada.

\section{Objetivos:}

Utilizar una historia clínica informatizada que garantice un manejo de datos sencillo, con acceso inmediato a toda información de un paciente y garantizando la confidencialidad. Optimizar recursos, fomentar y desarrollar el trabajo en equipo. Colaboración conjunta entre diferentes niveles asistenciales. Conseguir una atención integral y multidisciplinar, centrada en todos los procesos que afectan al paciente.

\section{Material y métodos:}

Utilización de programas de gestión de la historia clínica, por todos los profesionales implicados en el tratamiento y en todos los procesos que afecten al paciente. Utilización de todos los recursos que ofrecen estos programas para gestionar el tratamiento de los pacientes con enfermedad renal crónica.

\section{Resultados:}

Tras la implementación de estas herramientas en todos los centros que atienden a pacientes crónicos, se espera conseguir una visión global que permita una mejora en el manejo de la enfermedad, facilitando la comunicación entre profesionales y el acceso a los datos.

\section{Conclusiones:}

De todos estos motivos, se puede concluir que la historia clínica informatizada no solo es una herramienta útil, sino también necesaria y prácticamente imprescindible para abordar la ERC desde un punto de vista global que integre todo los procesos de salud-enfermedad de nuestros pacientes.

\section{Referencias Bibliográficas}

1. Manual de salud electrónica para directivos de servicios y sistemas de salud.

2. Manuales de la Historia Clinica Florence, utilizada en nuestro departamento. 